\title{
Article \\ A Morphable Physically Unclonable Function and True Random Number Generator Using a Commercial Magnetic Memory
}

\author{
Mohammad Nasim Imtiaz Khan ${ }^{1, *} \mathbb{*}$, Chak Yuen Cheng ${ }^{1,2}$, Sung Hao Lin ${ }^{1}$, Abdullah Ash-Saki ${ }^{1}$ (D) \\ and Swaroop Ghosh ${ }^{1}$ (D) \\ 1 Department of Electrical Engineering, Pennsylvania State University, State College, PA 16801, USA; \\ chakyuec@alumni.cmu.edu (C.Y.C.); frank19940124@gmail.com (S.H.L.); axs1251@psu.edu (A.A.-S.); \\ szg212@psu.edu (S.G.) \\ 2 Department of Electrical and Computer Engineering, Carnegie Mellon University, Pittsburgh, PA 15213, USA \\ * Correspondence: mohammad.nasim.imtiaz.khan@intel.com
}

check for

updates

Citation: Khan, M.N.I.; Cheng, C.Y.; Lin, S.H.; Ash-Saki, A.; Ghosh, S. A Morphable Physically Unclonable Function and True Random Number Generator Using a Commercial Magnetic Memory. J. Low Power Electron. Appl. 2021, 11, 5. https:// doi.org/10.3390/jlpea11010005

Received: 23 December 2020 Accepted: 11 January 2021 Published: 14 January 2021

Publisher's Note: MDPI stays neutral with regard to jurisdictional clai$\mathrm{ms}$ in published maps and institutional affiliations.

Copyright: $(\odot 2021$ by the authors. Licensee MDPI, Basel, Switzerland. This article is an open access article distributed under the terms and conditions of the Creative Commons Attribution (CC BY) license (https:// creativecommons.org/licenses/by/ $4.0 /)$.

\begin{abstract}
We use commercial magnetic memory to realize morphable security primitives, a Physically Unclonable Function (PUF) and a True Random Number Generator (TRNG). The PUF realized by manipulating the write time and the TRNG is realized by tweaking the number of write pulses. Our analysis indicates that more than $75 \%$ bits in the PUF are unusable without any correction due to their inability to exhibit any randomness. We exploit temporal randomness of working columns to fix the unusable columns and write latency to fix the unusable rows during the enrollment. The intra-HD, inter-HD, energy, bandwidth and area of the proposed PUF are found to be $0,46.25 \%$, $0.14 \mathrm{pJ} / \mathrm{bit}, 0.34 \mathrm{Gbit} / \mathrm{s}$ and $0.385 \mu \mathrm{m}^{2} /$ bit (including peripherals) respectively. The proposed TRNG provides all possible outcomes with a standard deviation of 0.0062 , correlation coefficient of 0.05 and an entropy of 0.95 . The energy, bandwidth and area of the proposed TRNG is found to be $0.41 \mathrm{pJ} / \mathrm{bit}$, $0.12 \mathrm{Gbit} / \mathrm{s}$ and $0.769 \mu \mathrm{m}^{2} /$ bit (including peripherals). The performance of the proposed TRNG has also been tested with NIST test suite. The proposed designs are compared with other magnetic PUFs and TRNGs from other literature.
\end{abstract}

Keywords: MRAM; TRNG; PUF; morphable security primitive; hardware security primitive

\section{Introduction}

There has been a proliferation of Internet-of-Things (IoTs) edge devices, and cybersecurity aspects of such devices are becoming a concern. Cybersecurity techniques, securing only the upper layer of software stack, are not sufficient anymore as underlying hardware faces a plethora of security and trust issues such as cloning, reverse engineering, Trojan insertion, side channel attack [1], recycling/counterfeiting, and so on. Therefore, many techniques and countermeasures are explored to ensure security and trust of hardware systems at various levels. For example, security primitives like recycling sensor [2], Physically Unclonable Functions (PUF) [3,4], True Random Number Generator (TRNG) [5], tamper sensor [6], encryption engines [7], Trojan detection [8-10], etc., are proposed to secure hardware. The security solutions are mostly driven by CMOS -based technologies. However, the CMOS-based solutions can be limited by the small set of features that can be leveraged to develop security primitives such as process-variation (PV) and thermal noise. In this regard, emerging technologies can be promising. They offer new sources of randomness and noise that can be harnessed to design robust security primitives. Besides, the solutions can achieve low power, high density, and high speed.

Prior Work on PUF: PUF is one of the widely accepted hardware security primitives that finds application in authentication. A PUF exploits differences between two chips due to intrinsic variation during the manufacturing process [4] to generate chip-specific and unique signatures. Several conventional and emerging technologies such as CMOS $[3,4]$, memristor [11] and spintronic technologies $[12,13]$ are explored to design PUFs. The CMOS 
PUFs include Static RAM (SRAM) based memory PUF, arbiter PUF and ring oscillator based PUFs [3]. Emerging technology based PUFs include memristor, spintronic memory, Resistive RAM (RRAM) [14,15], Domain Wall Memory (DWM), Magnetoresistive RAM (MRAM), etc. For example, DWM is used to design arbiter PUFs with exponential Challenge Response Pairs (CRP) which are resilient to machine learning attack [16]. Several PUFs based on Magnetoresistive RAM (MRAM) are also proposed [17-19]. In [17], the authors utilize unique energy-tilt of a Magnetic Tunnel Junction (MTJ) which stems from random geometric variations in the MRAM cells to generate PUF responses. The work in [18] identified the unreliable cells in a PUF to devise a zero bit-error-rate PUF. In Ref. [19], a strong PUF is proposed based on combining the resistances of a group of cells and generating their digital signature. The work exploits nano-scale analog disorders of MRAM, and this technique can be extended to other memory technologies.

Prior Work on TRNG: TRNGs exploit a source of randomness such as thermal noise, dynamic variations, etc. to generate random numbers. Ideally, the outputs of a TRNG must have high entropy and zero correlation. Several TRNGs are proposed using spintronic devices in prior work [20-23]. In [20,21], TRNG is implemented by manipulating the amplitude of the programming pulse. However, Ref. [20] requires controlling current in the order of $\mu$ As which is hard to achieve and Ref. [21] requires integration of analog circuit which is very sensitive to noise. A stochastic programming by current-driven STT using a Complementary Polarizer Spin Dice (CPSD) proposed in [22]. In [23], algorithms for PUF (based on read current) and TRNG (based on pulse width/amplitude manipulation) are proposed using MRAM. However, implementation details and results are not provided.

Proposed morphable PUF and TRNG: We propose a morphable security primitive using commercial magnetic RAM which can be used as both a PUF and a TRNG. To run it in the PUF mode, write time is controlled, and to run it in the TRNG mode, the number of write pulses is manipulated. Thus, it is named as morphable.

The magnetic tunnel junctions (MTJs) in the MRAM exhibit different write latencies owing to intrinsic and extrinsic PVs. For the same write time a bit may (or may not) flip in two different chips (extrinsic variation). This observation can be exploited to generate unique signatures from different chips which is useful for designing a PUF. We also notice that the same bit in a chip will randomly flip (intrinsic variation) if written multiple times with the same data. This is useful for designing a TRNG. The Figure 1 schematically shows the concept of re-purposing the MRAM in two different modes, i.e., PUF and TRNG. Thus, a $128 \mathrm{~KB}$ commercial MRAM chip can be converted to work solely as a $128 \mathrm{~KB}$ PUF or a 128 KB TRNG, or it can cohabitate a 64 KB PUF and a 64 KB TRNG.

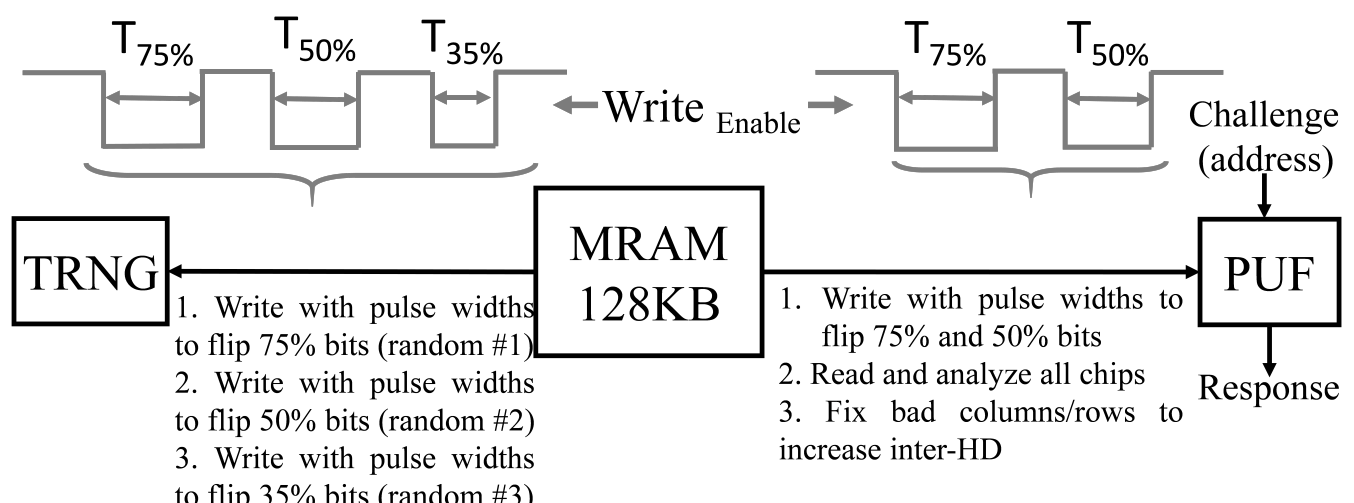

Figure 1. Morphable security primitive using Magnetoresistive RAM (MRAM). 
Note that the data width of the MRAM chips we used for this work is 8-bit. Therefore, we call each address as a row and number the rows with the corresponding address. Each row produces 8 bit and we number them as column 0 to 7 from Most Significant Bit (MSB) to Least Significant Bit (LSB). We summarize our methodology to realize PUF and TRNG from the MRAM chip below.

Working principle of proposed PUF (wPUF): First, we flush the bits of the PUF (write with 0s). Then, we try writing 1 in all the bits. The write time of the pulse is set to $50 \%$ switching probability so that $50 \%$ of the bits flip. However, due to stochastic nature of the bitcell and process variation, each chip will be written with different data which can be used as the signature of the chip. However, our analysis shows that 4 columns of all rows of the chips are stuck to 0 ( 2 columns)/1 ( 2 columns) and do not show probabilistic switching as expected. Remaining 4 columns show the probabilistic switching and therefore, overall switching probability is around 50\%. These severely limits PUF variation from chip to chip. We noted that the 4 columns are stuck because either they are very strong (stuck to 0 , requires more write time) or weak (stuck to 1 , requires less write time and always gets written to 1). We expect that the PUFs based on real memory chip implementation of any emerging NVMs might exhibit this type of behavior. Therefore, some of the bits of each address could be unusable for PUF. In this work, we propose techniques to fix these bad columns by exploiting the temporal randomness of good columns. Note that this is in contrast to MRAM and STT-MRAM PUFs presented in literature [17-19] that are specifically and carefully designed (bits, access transistor and peripherals) to amplify and capture the variability and to achieve high inter-HD and low intra-HD.

Working principle of proposed TRNG: Our analysis show that just biasing an address with $50 \%$ switching probability does not provide all possible outcomes. For example, the number of possible outcomes for a 4-bit TRNG is 16. However, we observed less number of outcomes due to strong/weak bits which limits the scope of the TRNG and makes it Pseudo Random Number Generator (PRNG). Therefore, we propose the following technique for TRNG: first, we write all $0 \mathrm{~s}$ in the cells of TRNG; then we write all bits to $1 \mathrm{~s}$ by selecting the write time to flip $75 \%$ of the bits (i.e., $75 \%$ switching probability) to extract the first random number. For generating the second and third random number from the same address, we propose to repeat the above steps with the write time to flip $50 \%$ of the bits (i.e., $50 \%$ switching probability) and with the write time to flip $25 \%$ of the bits (i.e., $25 \%$ switching probability) respectively. This way we get all 16 possible outcomes from the 4 good columns with tolerable standard deviation. For example, $75 \%$ switching probability will mainly generate $4^{\prime} \mathrm{b} 0111,4^{\prime} \mathrm{b} 1011,4^{\prime} \mathrm{b} 1101$ and $4^{\prime} \mathrm{b} 1110,50 \%$ will mainly generate $4^{\prime} \mathrm{b} 0011,4^{\prime} \mathrm{b} 0101,4^{\prime} \mathrm{b} 1001,4^{\prime} \mathrm{b} 0110,4^{\prime} \mathrm{b} 1010$ and $4^{\prime} \mathrm{b} 1100$ and 25\% will mainly generate $4^{\prime} \mathrm{b} 0001,4^{\prime} \mathrm{b} 0010,4^{\prime} \mathrm{b} 0100$ and $4^{\prime} \mathrm{b} 1000$. The remaining ones $\left(4^{\prime} \mathrm{b} 0000 / 4^{\prime} \mathrm{b} 1111\right)$ are also generated (with lower recurrence number) mainly with $25 \% / 75 \%$ probability if all the four bits are either very strong or weak respectively. Note that we did not fix the 4 bad columns with the good ones in case of TRNG. This is to prevent machine learning attacks that can profile the TRNG outcomes with fewer iterations (since the fixing can make the bits correlated).

Morphable Security Primitive: To use the MRAM in the TRNG mode (i.e., to generate unique true random numbers), the MRAM needs to be re-written every time. On the other hand, to use it as a PUF, the MRAM needs to be written only once (during the enrollment phase). The data that get written to PUF addresses depends on the PV which cannot be replicated by a malicious entity. It should be noted that PUF can be morphed to TRNG if written multiple times as proposed for TRNG.

To the best of our knowledge, this is the first experimental demonstration of a PUF and TRNG using commercial MRAM chip. A work-in-progress version of this work has been published in [24] where the methodology is discussed and initial data were presented. However, this work explains the design and results in detail. In summary, we make the following contributions: 
- We characterize the MRAM bit-to-bit write latency under voltage and temperature variations.

- We characterize the MRAM response under multiple write disturbs which can be useful for TRNG.

- We propose a write PUF (wPUF) by biasing the MRAM with a write latency with 50\% switching probability. The proposed PUF exhibits excellent stability and uniqueness.

- We show that $75 \%$ of the bits could be unresponsive to a challenge and propose techniques to convert them into useful bits avoiding expensive row and columns masking.

- We propose a TRNG by exploiting random MRAM responses under multiple write disturbs.

- We benchmark the proposed PUF and TRNG with existing designs.

The rest of the paper is organized as follows: Section 2 provides the background of MRAM technology, details of the experimental setup and analysis of the MRAM responses to write latency and number of writes. Sections 3 and 4 describe the proposed PUF and TRNG. Section 5 presents discussion and Section 6 draws conclusions.

\section{Background on MRAM and Its Variation}

In this section, we present basics of the toggle MRAM and characterize its statistical and temporal behavior.

\subsection{Basics of MRAM}

MRAM bitcell (Figure 2) contains one MTJ and one NMOS access transistor. The MTJ lies between a pair of metal-lines named digit-line and bit-line to facilitate write operation. The metal-lines are parallel to the cell plane and placed orthogonal to each other. An induced magnetic field is created with appropriate polarity by passing current through the lines during write operation. The field exerts a torque on the free layer magnetic orientation, causing it to flip. During read operation, the access transistor is turned ON, and a fixed voltage is applied across the cell to sense the equivalent resistance.

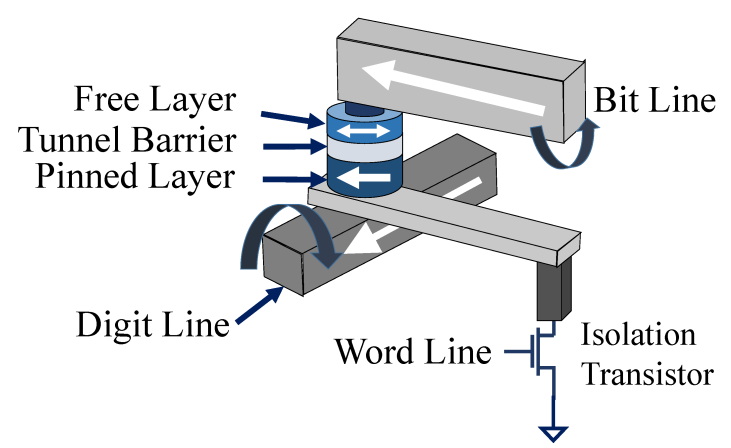

Figure 2. Toggle MRAM bitcell containing one Magnetic Tunnel Junction (MTJ) and one access transistor.

A commercial toggle MRAM chip (MR4A08B) with 2 million rows and 8 columns is used in this work to validate the proposed morphable security primitive. Table 1 captures the key features of the MRAM chip. It should be noted that we have used the first $128 \mathrm{~KB}$ (1 Mbit) out of $16 \mathrm{Mbit}$ of the chip. Figures 3 and 4 presents the timing diagram of the write and read operations of the MRAM chip. From the data sheet, it is evident that the chip requires maximum of $15 \mathrm{~ns}$ of write enable (low) signal for successful write and data is valid after $25 \mathrm{~ns}$ of read enable signal. In this work, we modified the specified read and write times to realize the proposed PUF and TRNG. 
Table 1. Characteristic of the MRAM Chip.

\begin{tabular}{cc}
\hline Parameter & Value \\
\hline Capacity & $16 \mathrm{Mbit}$ \\
Read/Write Cycle & $35 \mathrm{~ns}$ \\
Address/Data Bus Length & $21 / 8$ \\
Retention Time & $>20$ years \\
AC stand by Current & $9-14 \mathrm{~mA}$ \\
AC Active Current (Read/Write) & $60-68 \mathrm{~mA} / 152-180 \mathrm{~mA}$ \\
\hline
\end{tabular}

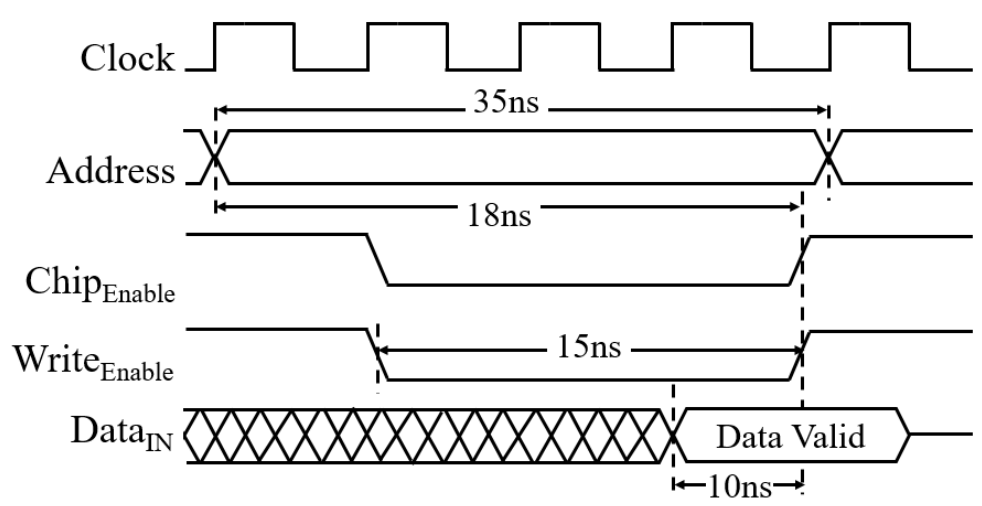

Figure 3. Write cycle of MRAM chip. Note that the chip requires maximum of $15 \mathrm{~ns}$ of write enable (low) signal for successful write.

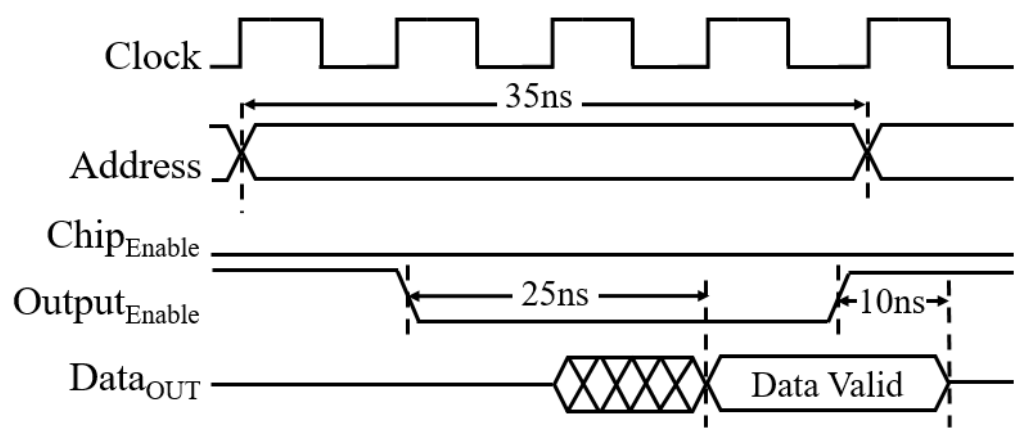

Figure 4. Read cycle of MRAM chip.

\subsection{Experimental Setup}

A four-layer PCB is designed to interface the MRAM chip with a logic analyzer and Digilent Basys3 [25]. The PCB is designed with minimum wire resistance, inductance and capacitance to stabilize the supply current for PUF power measurement. A $1 \Omega$ thick film current sensing resistor is connected between the ground of the PCB and the MRAM chip to sense the MRAM current during read/write. A heat gun is used to change the ambient temperature. We have analyzed a total of 10 chips.

First, we interfaced the MRAM chip with logic analyzer and wrote all addresses at faster than 15 ns write time@ $V_{D D}=3.3 \mathrm{~V}, 25^{\circ} \mathrm{C}$. We found that the data is written successfully for all chips at 12 ns (Figure 5). Therefore, the write time should be less than $12 \mathrm{~ns}$ at nominal operating condition to bias the cells with $50 \%$ write probability. We read the data at $30 \mathrm{~ns}$ in order to avoid read failures. Next, we interfaced the MRAM chip with the FPGA. The writing is done at much higher frequency to achieve partial write and reading is done at $25 \mathrm{MHz}(40 \mathrm{~ns})$. The read/write traces are captured by Keysight DSOS-804A Oscilloscope [26] with sampling frequency of $20 \mathrm{GSa} / \mathrm{s}$ and Bandwidth of $8 \mathrm{GHz}$. The experimental setup is shown in Figure 6. Data is sent to a PC using UART (baud rate 9600 bps) for MATLAB analysis. 

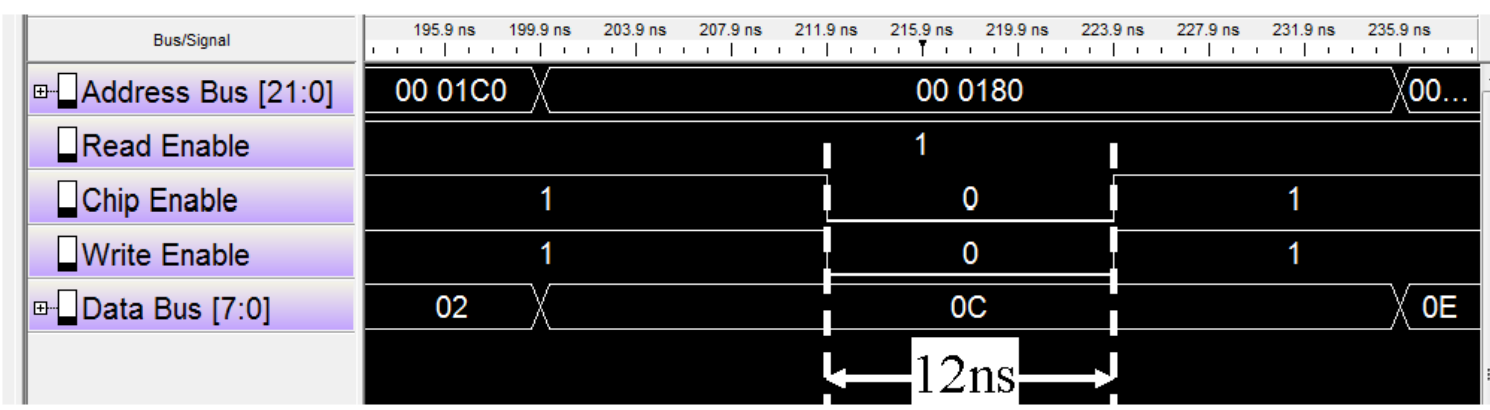

(a)

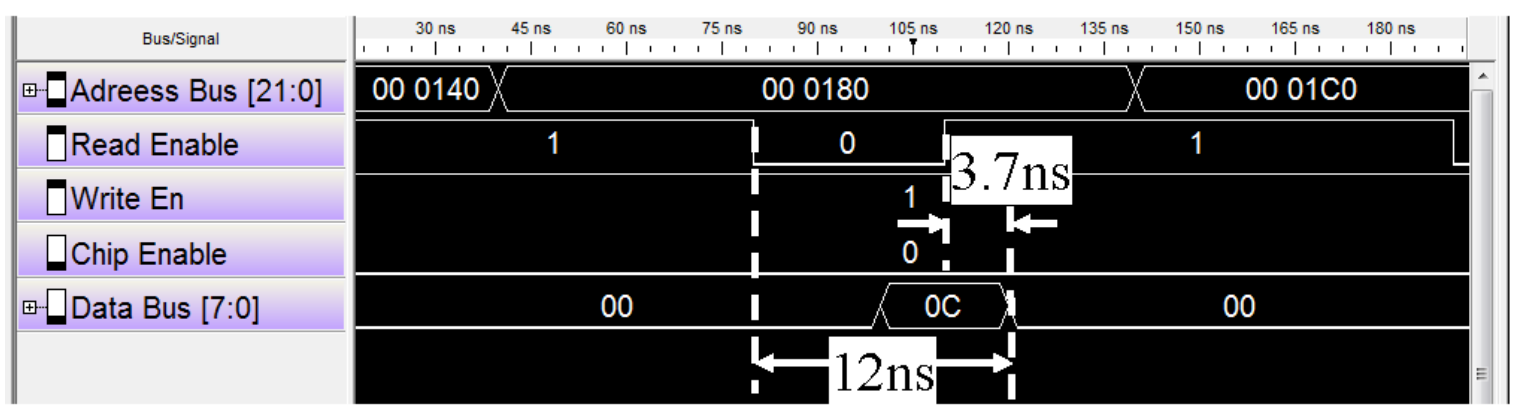

(b)

Figure 5. MRAM (a) write and (b) read waveforms. Note that with 12 ns of write enable (low) signal, data (0x0C) is written successfully (to address 0x00 0180).

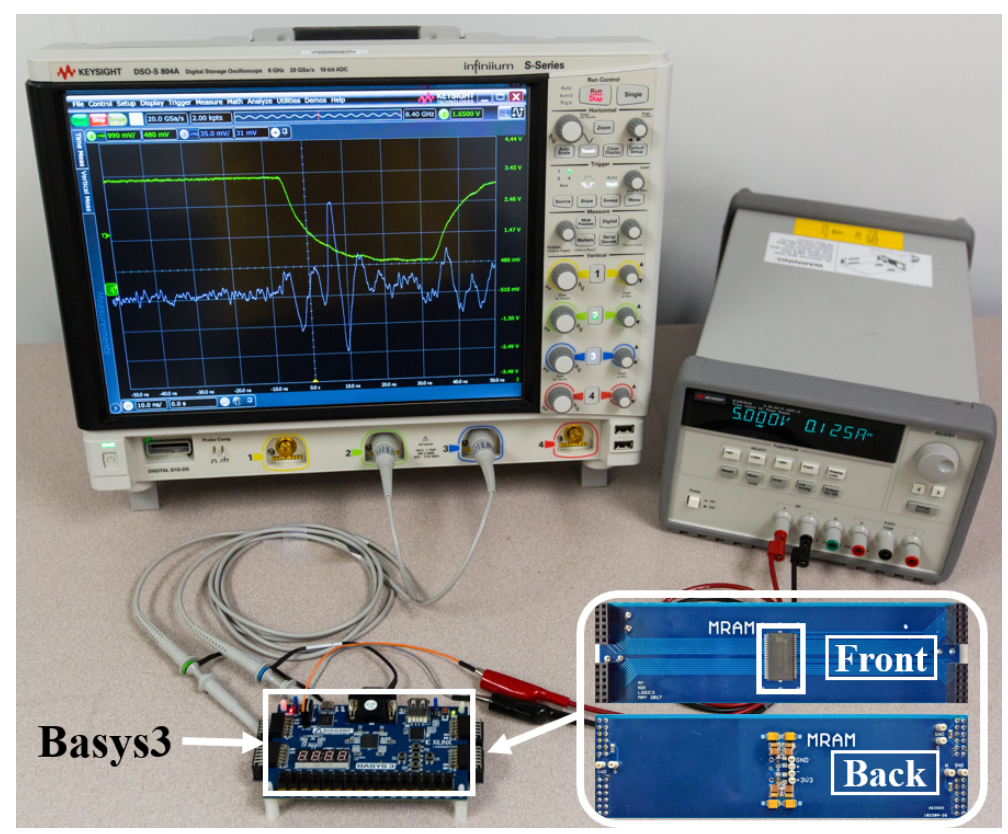

Figure 6. Experimental setup. The MRAM PCB (inset) is mounted on top of Basys3 for noise reduction.

\subsection{Switching Variation of MRAM}

We swept the write time of each of the 10 chips to identify the Cumulative Distribution Function (CDF) of write time for $0 \rightarrow 1$ switching. Figure 7 shows the CDFs of three chips that are found to possess highest/median/lowest write times. To realize PUF, the write latency of each chip should be chosen to write $50 \%$ of the bits to get uniform $0 / 1$ distribution. From Figure 7 it is obvious that write time varies from chip to chip. This could be useful for TRNG application. From the experimental results and analysis, we note the following: 
(a) The write latency can be arranged in increasing order as, $0 \rightarrow 0,1 \rightarrow 0,0 \rightarrow 1$, $1 \rightarrow 1$ (i.e., writing $0 \rightarrow 0$ takes shortest time and $1 \rightarrow 1$ takes longest time $\left.\left(T_{(1 \rightarrow 1)}\right)\right)$.

(b) For writing $1 \rightarrow 1$ with $t_{\text {write }}<10 \mathrm{~ns}$ and $10 \mathrm{~ns}<t_{\text {write }}<T_{1 \rightarrow 1}$, the stored data becomes 0 and stochastic respectively. From this observation we conclude that the chip initializes the data to 0 before writing a 0 or 1 . We believe that, this observation is rooted at the toggle MRAM implementation which toggles the bit (irrespective of the existing state) during write.

(c) Consecutive writes of $1 \mathrm{~s}$ with the same short write time reduces number of $1 \mathrm{~s}$ in the data (consequence of (b)). For example, if the addresses are flushed with all $0 \mathrm{~s}$ and then written with all $1 \mathrm{~s}$ with write time to flip $75 \%$ bits (which is $<T_{1 \rightarrow 1}$ ) and the writing is repeated with same time (without flushing in between), the number of $1 \mathrm{~s}$ in the data reduces to $\approx 49 \%, \approx 34 \%$ and then remains fairly constant (Figure 8 ). This can be useful for TRNG (further explained in Section 4).

(d) Columns 0 and 7 are biased to data 0 , columns 1 and 2 are biased to data 1 and column 3-6 mainly shows 50\% switching probability (Figure 9) although we select the write time to flip $50 \%$ of the bits for the entire memory array. Therefore, columns $0,1,2$ and 7 cannot be used for PUF and TRNG. If every cell needs to be biased at their own 50\% switching probability, then write time for each cell in a row/column needs to be controlled individually which is practically impossible.

(e) Error-free read can be performed with 23.3 ns under 3.0 V-3.6 V and 25 C-60 C. The highest read latency is $23.3 \mathrm{~ns} @ 3.0 \mathrm{~V}, 60 \mathrm{C}$ and lowest read latency is $22.47 \mathrm{~ns} @ 3.6 \mathrm{~V}$, $25 \mathrm{C}$.

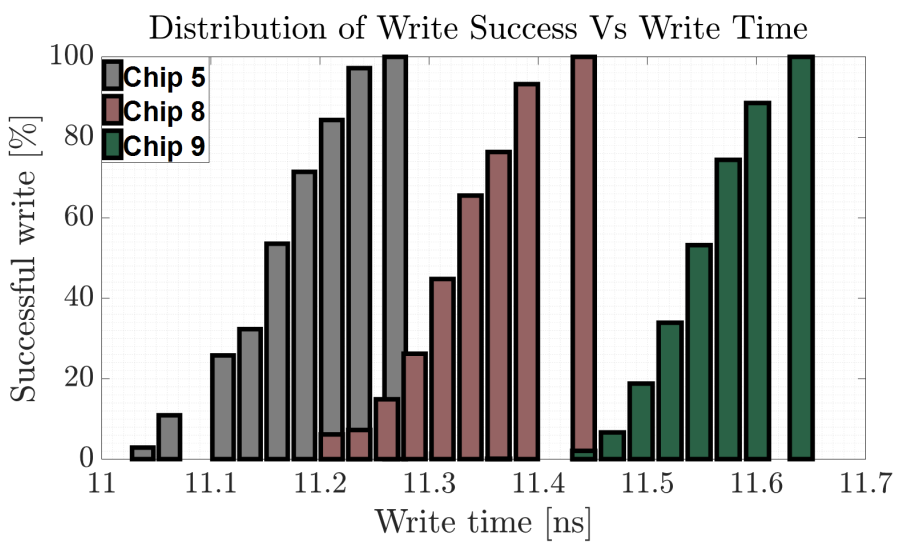

Figure 7. Cumulative Distribution Function (CDF) of write success of 3 chips (out of 10). These 3 chips exhibit maximum write time variation.

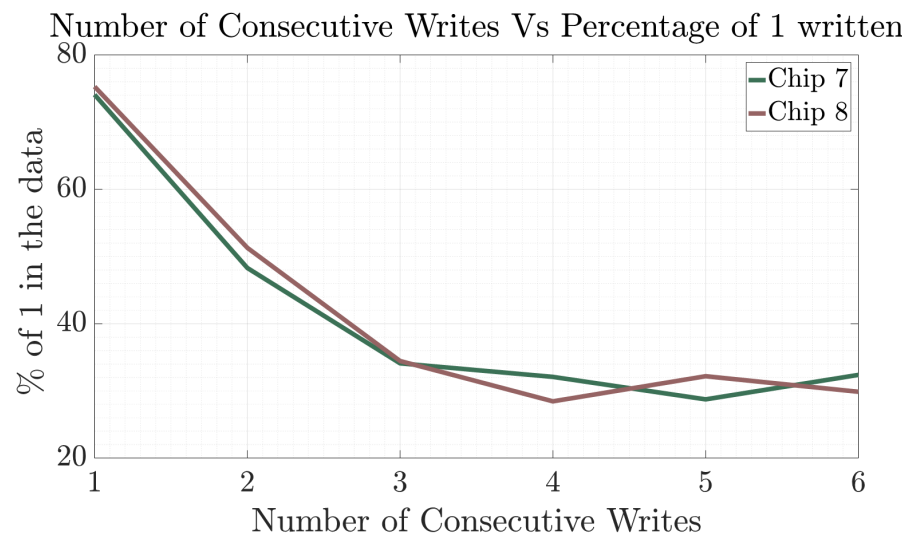

Figure 8. Number of consecutive writes vs. percentage of $1 \mathrm{~s}$ in the data. 


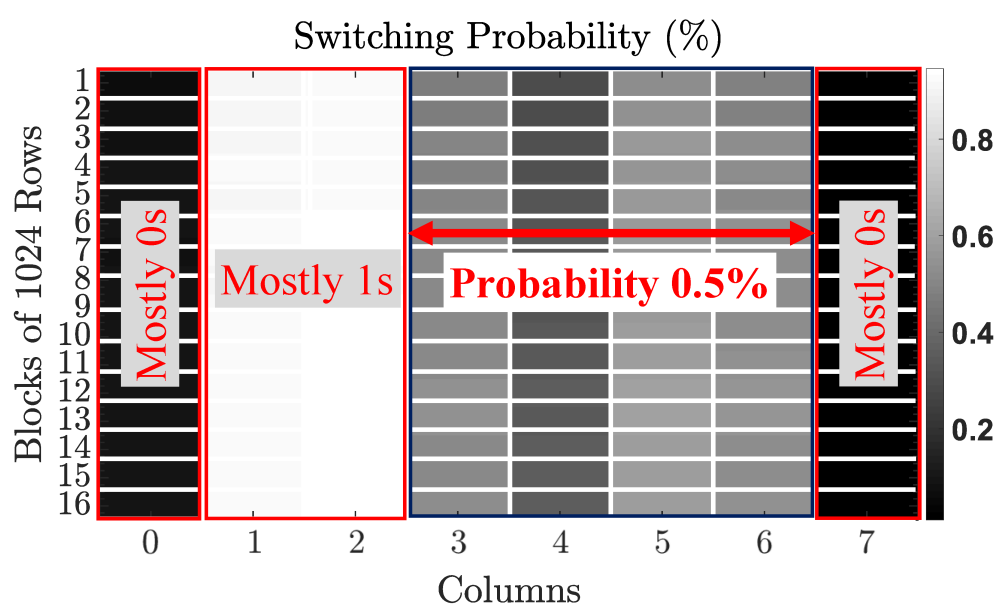

Figure 9. Switching probability distribution of 16 KB MRAM. First 16384 rows are grouped to 16 blocks (1024 rows/block).

\section{PUF}

Memory PUFs exploit random initialization of the memory bits due to PV. The address bits are used as challenge and the bits read from the memory array with the address is the response of the PUF. In this section, we present the proposed wPUF and analyze its performance using experimental results.

\subsection{Proposed wPUF}

The principle of the proposed wPUF is explained in algorithm (Figure 10). The wPUF will have two phases:

\section{Enrollment (Phase 1)}

Flush all addresses @ rated write time

\begin{tabular}{c}
$\begin{array}{c}\text { Find write time to switch 50\% bits }\left(\mathrm{T}_{50 \%}\right) \text { for } \\
\text { the chip @ controlled temperature and } \mathrm{V}_{\mathrm{DD}}\end{array}$ \\
$\begin{array}{c}\text { Read data @ rated read time, fix bad } \\
\text { columns and rows (or blocks of rows) for } \\
\text { maximizing inter-HD }\end{array}$ \\
\hline$\downarrow$ \\
Write the responses back
\end{tabular}

(a) Enrollment phase

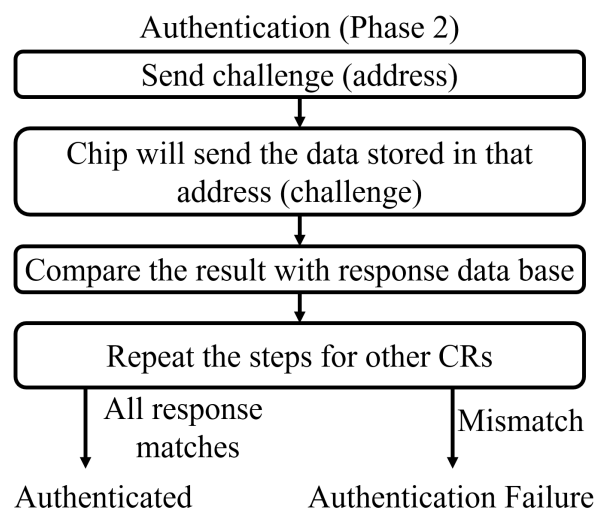

(b) Authentication phase

Figure 10. Two phases of wPUF, (a) Enrollment phase; (b) Authentication phase.

(a) Enrollment phase (Figure 10a): In this phase, unique signature from a chip is extracted using the method described below. First, the manufacturer prepares the memory in an initial state by writing all bits with $0 \mathrm{~s}$ (flushing of the MRAM). Next, the manufacturer try writing all $1 \mathrm{~s}$ with a write time $T_{50 \%}$ to switch $50 \%$ bits to 1 . Note that, $T_{50 \%}$ is supply voltage $\left(V_{D D}\right)$ and temperature dependent. As the manufacturer has access to a controlledenvironment, managing target $V_{D D}$ and temperature will not be an issue. Finally, the entire address space is read to extract unique signatures. The steps are repeated for several chips to create a pool of data for statistical analysis and subsequent correctional measures. Then, all chip responses will be compared to separate the unresponsive columns/rows (that show no variation) for all chips and which gives highest uniqueness for different chips. Based on this observation, the unresponsive columns and rows need further processing (discussed in Section 3.3). 
(b) Authentication phase (Figure 10b): This phase involves sending challenges (memory addresses) to the PUF and getting back responses (data stored in those addresses). Sufficient number of CRPs should be matched so that a chip can be distinguished as unique and authenticate. If there are reasonable mismatch in the CRP pairs, authentication should fail.

It should be noted that the enrollment phase for a chip is just a one-time operation. Once enrollment phase is performed by the manufacturer, only authentication phase (involves read operation) needs to be performed in real time.

\subsection{Performance Analysis}

PUF performance is evaluated with mainly three parameters:

\subsubsection{Uniqueness (Inter-Die HD)}

Uniqueness (measured by inter-die HD) in the PUF response enables the identification of different chips uniquely. A 50\% inter-die HD is desirable. In this work, the inter-die $\mathrm{HD}$ is calculated for 10 chips and the average is found to be only $22.5 \%$ which is very low. The reason for getting the low inter-HD is attributed to, (i) a smaller number of bits per challenge ( 4 bits) to compare the responses of different chips (only 16 combinations), and, (ii) inability to bias each bit at their $50 \%$ switching probability individually. The inter-die HD is improved in the next subsection.

\subsubsection{Reliability (Intra-Die HD)}

Reliability (measured by intra-die HD) is the measure of the dependency of PUF response to the intra chip voltage and temperature variations. A $0 \%$ intra-die HD is desirable. Intra-die HD is measured by XORing the responses of the PUF at various voltages and temperatures. In this work, we capture the responses of all chips for $V_{D D}$ ranging $3.0 \mathrm{~V}$ to $3.6 \mathrm{~V}$ and temperature ranging from $25^{\circ} \mathrm{C}$ to $60^{\circ} \mathrm{C}$ with read time of $23.3 \mathrm{~ns}$ which gives intra-die $\mathrm{HD}=0 \%$. A perfect intra-die $\mathrm{HD}$ is achieved by relaxing the read time. System throughput can be increased by reducing the read time which incurs a non-zero intra-die HD for some operation condition. We propose to implement relaxed read time and compromise system throughput since achieving $0 \%$ intra-die HD is critical.

\subsubsection{Uniformity}

For uniformity in the PUF response, the probability of 1's and 0's in the response for possible challenges should be $50 \%$. We evaluate the uniformity by the frequency metric in the NIST benchmark for all the possible 16 CRPs of 4 MRAM bits of the PUF and found it to be $\approx 48 \%$ with block frequency test. Entropy test on the responses show satisfactory $p$-value (>0.01) which ensures randomness.

\subsection{Improving Inter-HD}

Figure 11 shows the average inter-HD of all 10 chips for first 80 rows ( 8 rows per row block). From the result, we observe that inter-HD of Col 0, 1, 2 and 7 are poor as they are stuck to either 0 or 1 . Furthermore, we also observe that certain address rows provide good inter-HD (green/blue) whereas other rows provide very poor inter-HD (red). We propose the following techniques to improve the inter-HD.

\subsubsection{Improving Column Performance}

We propose writing the same address twice with write time corresponding to $75 \%$ switching probability. The first/second response will have $75 \% / 50 \%$ number of 1 s. Since the two responses are purely random, we propose using first response of columns 3-6 as the response of bad columns (i.e., columns 0,1,2 and 7) and the second response of columns 3-6 as their own response. Therefore, we avoid masking of unusable columns and restore the PUF bandwidth to 8 bits per challenge. The response obtained for bad columns are enforced on them with relaxed write latency for subsequent authentication. 
This technique exploits the fact that each bit of MRAM can produce more than one random bit of information.

\subsubsection{Improving Row Performance}

By improving column performance, inter-die HD of stuck columns improves by $1.7 \mathrm{X}$ $4 X$ (individually). However, total inter-HD remains poor $(\approx 28 \%)$ due to poor performance of some rows that exhibit $0 \%$ inter-HD. Further, we observe that the rows with poor inter-HD are mainly stuck to either all 0 s or all 1s. The rows stuck to 0s (perhaps due to higher thermal stability) are fixed by re-writing them with higher write time to trigger statistical flipping. The row stuck to all 1s (perhaps due to low thermal stability) are fixed by re-writing them with lower write time.

After improving row/column performances, we obtain inter-HD of $46.25 \%$ which is close to the ideal value. The proposed improvement technique incurs one-time energy $(\approx 2.7 \mathrm{X})$ and computational overhead during the enrollment phase. Furthermore, it should be noted that, alternative improvement technique of masking the rows/blocks of rows incurs significant area overhead. For the case of Figure 11,75\% bits are lost if masking is implemented. The energy, bandwidth and area of the wPUF are $0.14 \mathrm{pJ} / \mathrm{bit}, 0.34 \mathrm{Gbit} / \mathrm{s}$, $0.385 \mu \mathrm{m}^{2} /$ bit (including all peripheral circuits). Table 2 benchmarks proposed wPUF with existing MRAM/STTRAM PUFs. We can observe that the proposed PUF is comparable to prior works. It should be noted that (i) the proposed PUF is based on a commercial chip which gives practical process variation; and, (ii) the data bus is 8 bit long, therefore the bandwidth is comparatively low.

\begin{tabular}{|c|c|c|c|c|c|c|c|c|c|}
\hline & \multicolumn{8}{|c|}{ Column } \\
\hline & & 1 & 2 & 3 & 4 & 5 & 6 & 7 & 8 \\
\hline \multirow{10}{*}{ 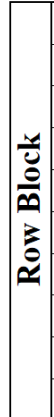 } & 1 & 0.05 & 0.125 & 0.125 & 0.275 & 0.25 & 0.25 & 0.25 & 0 \\
\hline & 2 & 0.05 & 0.125 & 0.15 & 0.35 & 0.3 & 0.45 & 0.475 & 0 \\
\hline & 3 & 0.05 & 0.125 & 0.125 & 0.35 & 0.275 & 0.375 & 0.35 & 0 \\
\hline & 4 & 0.125 & 0.075 & 0.075 & 0.05 & 0.25 & 0.05 & 0.1 & 0.1 \\
\hline & 5 & 0.05 & 0.375 & 0.375 & 0.225 & 0.15 & 0.35 & 0.25 & 0 \\
\hline & 6 & 0.05 & 0.15 & 0.15 & 0.425 & 0.375 & 0.475 & 0.45 & 0 \\
\hline & 7 & 0.05 & 0.15 & 0.15 & 0.475 & 0.35 & 0.475 & 0.325 & 0 \\
\hline & 8 & 0.125 & 0.125 & 0.125 & 0.175 & 0.225 & 0.05 & 0.175 & 0.075 \\
\hline & 9 & 0 & 0.175 & 0.175 & 0.2 & 0.125 & 0.35 & 0.125 & 0 \\
\hline & 10 & 0.05 & 0.05 & 0.05 & 0.425 & 0.275 & 0.475 & 0.4 & 0 \\
\hline
\end{tabular}

Figure 11. Average inter-HD of 10 chips for first 80 rows (8 rows/block).

Table 2. Performance comparison of different MRAM/STTRAM PUFs with the proposed wPUF.

\begin{tabular}{cccccccc}
\hline PUFs & $\begin{array}{c}\text { Inter-Die HD } \\
(\mathbf{\%})\end{array}$ & $\begin{array}{c}\text { Inter-Die HD } \\
(\mathbf{\%})\end{array}$ & Entropy & $\begin{array}{c}\text { Area }(\mathbf{M T J}) \\
\left(\mu \mathbf{m}^{\mathbf{2}}\right)\end{array}$ & $\begin{array}{c}\text { Bandwidth } \\
(\mathbf{G b i t} / \mathbf{s})\end{array}$ & $\begin{array}{c}\text { Energy/bit } \\
(\mathbf{p J})\end{array}$ & Experimental \\
\hline$[15]$ & - & 50.1 & 0.985 & 0.046 & 6.4 & - & No \\
\hline$[17]$ & 0.02 & 47 & 0.99 & $\begin{array}{c}6.74 \\
(64 \mathrm{bit})\end{array}$ & 12.8 & - & Yes \\
\hline$[27]$ & 7.76 & 60.6 & - & 0.065 & 6.4 & 2.42 & No \\
\hline$[28]$ & - & 49.89 & 0.95 & 0.005 & 6.4 & 0.001 & No \\
\hline $\begin{array}{c}\text { wPUF } \\
\text { (This Work) }\end{array}$ & 0 & $\begin{array}{c}22.5 \text { (before) } \\
46.26 \text { (after) }\end{array}$ & 0.95 & $0.385^{1}$ & 0.34 & $0.14^{1}$ & Yes \\
\hline
\end{tabular}

${ }^{1}$ includes decoder, sense amp, and other peripheral circuitry and considers $100 \mathrm{mil} \times 100 \mathrm{mil}$ of die size for TSOP-II IC package. 


\section{TRNG}

TRNG generates true random number based on some random inherent noise. For memory-based TRNG, the memory bitcell is biased at the 50\% switching probability point at which the bit stabilizes to either 1 or 0 depending on the noise. Therefore, the written value is highly unpredictable, varies from chip to chip and strongly depends on the operating conditions and noise. In this section, we present the proposed TRNG and analyze its performance using experimental results.

\subsection{Proposed TRNG}

Figure 12 presents the algorithm to realize TRNG from the MRAM chip. First, the memory address is flushed, i.e., $0 \mathrm{~s}$ are written to all bits. Next, the write time is set to $75 \%$ switching probability and $1 \mathrm{~s}$ are written to the addresses. The $75 \%$ switching probability means the ratio of $1 \mathrm{~s}$ to $0 \mathrm{~s}$ will be $75 \%$. The data written is considered as the first random number. Then, the same address is written again with all $1 \mathrm{~s}$ with the same write time. We notice that the ratio of $1 \mathrm{~s}$ to $0 \mathrm{~s}$ reduces to $\approx 50 \%$ (observation (b), Section 2) on the second write. This is the second random number. Similarly, when we write for the 3 rd time the $1 \mathrm{~s}$ to $0 \mathrm{~s}$ ratio drops to $\approx 35 \%$ which gives out the 3 rd random number. By repeating the same process for different write times and different addresses, we can generate more random numbers.

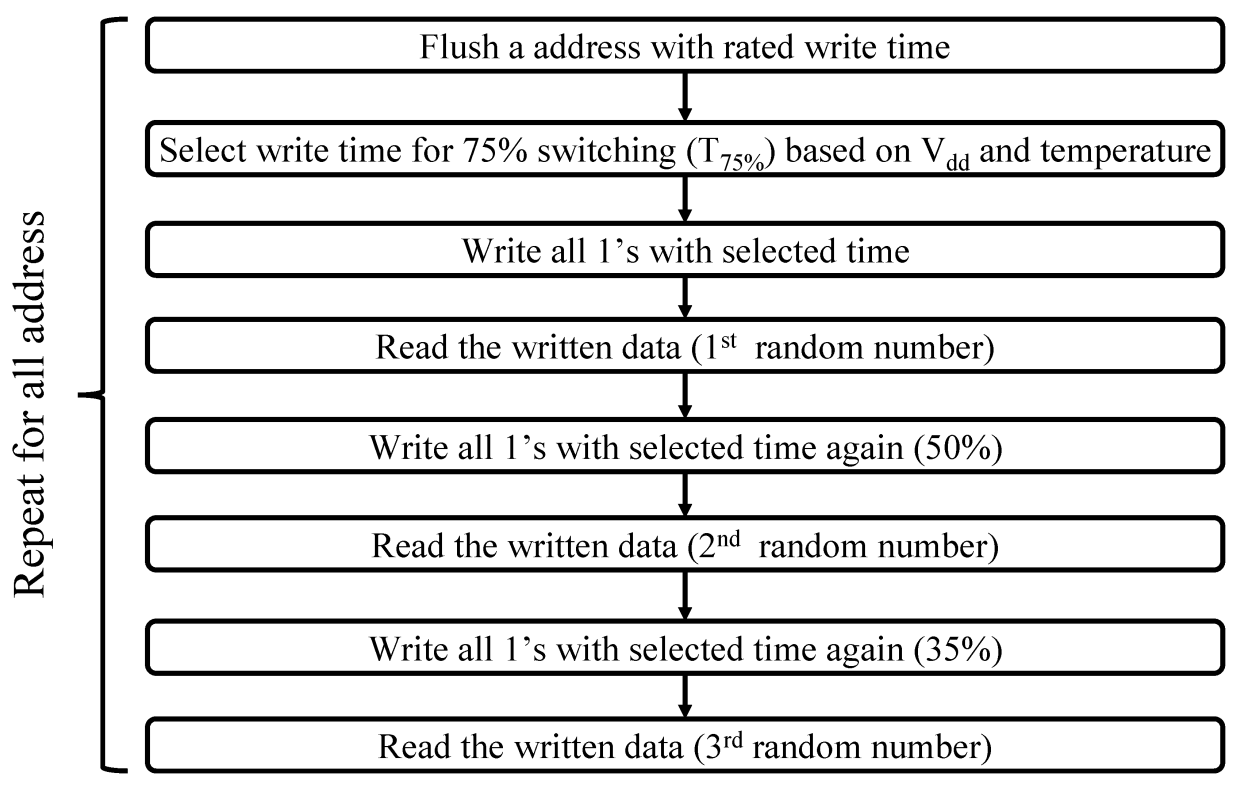

Figure 12. Algorithm of the proposed True Random Number Generator (TRNG).

Since it is impossible to bias individual MRAM bits to their $50 \%$ switching probability using a common write latency, we bias the MRAM using three different switching probabilities and extract all possible outcomes from n-bits. For example, biasing 4 bits at $50 \%$ switching probability means that on an average, 2 bits out of 4 will be set to ' 1 '. This will produce 6 outcomes out of 16 possibilities (i.e., 1100, 0110, 0011, 1010, 1001 and 0101). Biasing at $75 \%$ switching probabilities mean 3 bits will be set to 1 s producing 4 new outcomes (1110, 0111, 1011 and 1101). Biasing at 35\% switching probabilities mean 1 bit will be set to 1 s producing 4 new outcomes $(0001,0010,0100$ and 1000). Note that the remaining two outcomes $(0000 / 1111)$ are also produced by TRNG but with lower frequency.

Write operation of any spintronic memory suffers due to temperature and $V_{D D}$ variation. Therefore, TRNG can be biased to some preferential values and behaves as Pseudo Random Number Generator (PRNG). Tracking of $V_{D D}$ and temperature are needed for magnetic TRNG to change the biasing conditions accordingly and achieve desired switching probability. 
We assume that the processor can track $V_{D D}$ and temperature to select appropriate write time. Figure 13a shows the proposed circuit and Figure $13 \mathrm{~b}$ shows the timing waveform to implement variable write time. For this example, we have assumed that the processor runs at $1 \mathrm{GHz}$ (time period $1 \mathrm{~ns}$ ) and write time can vary from 9 ns to $13 \mathrm{~ns}$ depending on operating condition. Therefore, from this circuit we can select write time with a step size of $0.5 \mathrm{~ns}$ in that range (by asserting WR_ENABLE and any of the other 8 pulses). If finer step size is required, more pulses can be generated with less duty cycle. However, in that case, the MUX will have more inputs (4:1 MUX (16 pulses in total) for $0.25 \mathrm{~ns}$ granularity).

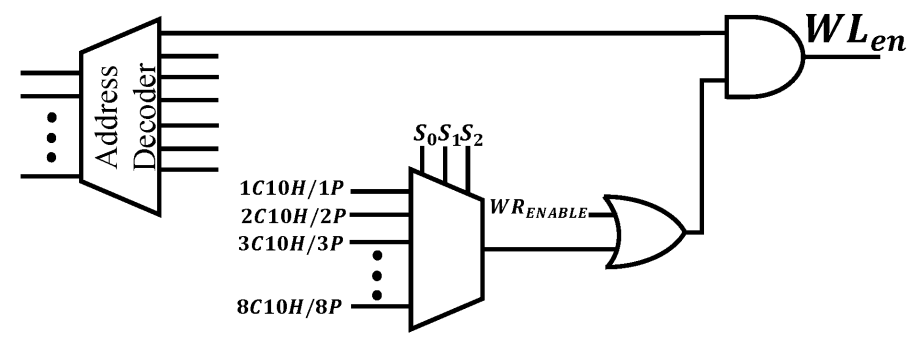

(a)

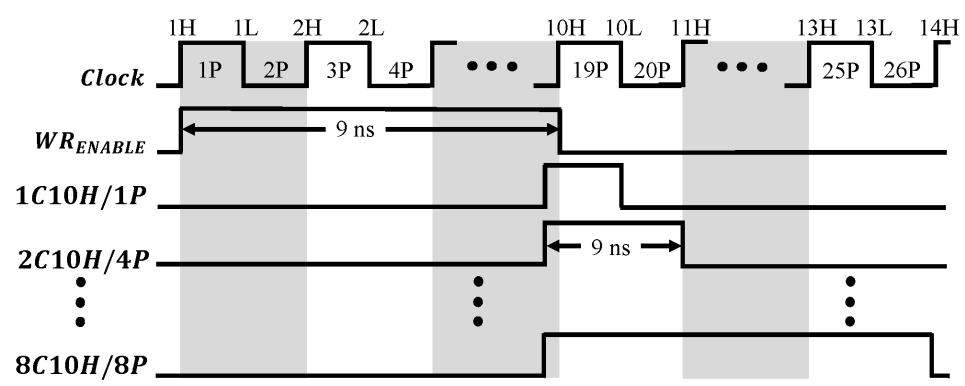

(b)

Figure 13. (a) Circuit and (b) timing waveform to implement specific write time selection for achieving desired switching probability.

\subsection{Performance Analysis}

(a) Entropy: Entropy of TRNG defines the randomness of the generated data. Entropy can be calculated with the following equation [29]:

$$
E=-\sum_{i=2}^{2} p_{i} \log _{2}\left(p_{i}\right)
$$

where $\mathrm{p} 1$ and $\mathrm{p} 2$ are probability of $1 \mathrm{~s}$ and $0 \mathrm{~s}$ respectively in a n-bit long data stream. For an ideal TRNG, the entropy is 1 . We have calculated entropy of all chips column-wise and the average entropy is shown in Figure 14a. It is evident that, columns $0,1,2$, and 7 show poor randomness (explained in previous section) and therefore needs to be masked. After masking, the proposed TRNG offers an entropy of 0.95 .

(b) Repeatability: Repeatability is another important metric to evaluate TRNG performance. Ideally, a TRNG should only repeat when all other possible cases are already covered. For example, for a 4-bit TRNG, the generated value should only repeat when all the 16 possible values are generated. However, practically this is very difficult to achieve. In the proposed TRNG algorithm, we are able to get all possible outcomes with small standard deviation (0.0062) and the correlation coefficient is calculated as 0.05 . Figure $14 \mathrm{~b}$ shows the outcomes of 10,000 cases for the proposed TRNG. 


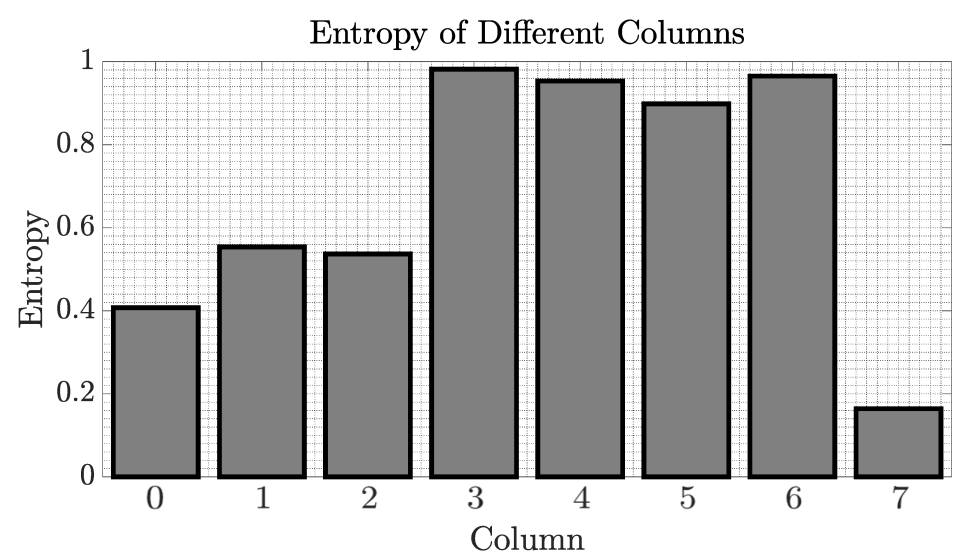

(a)

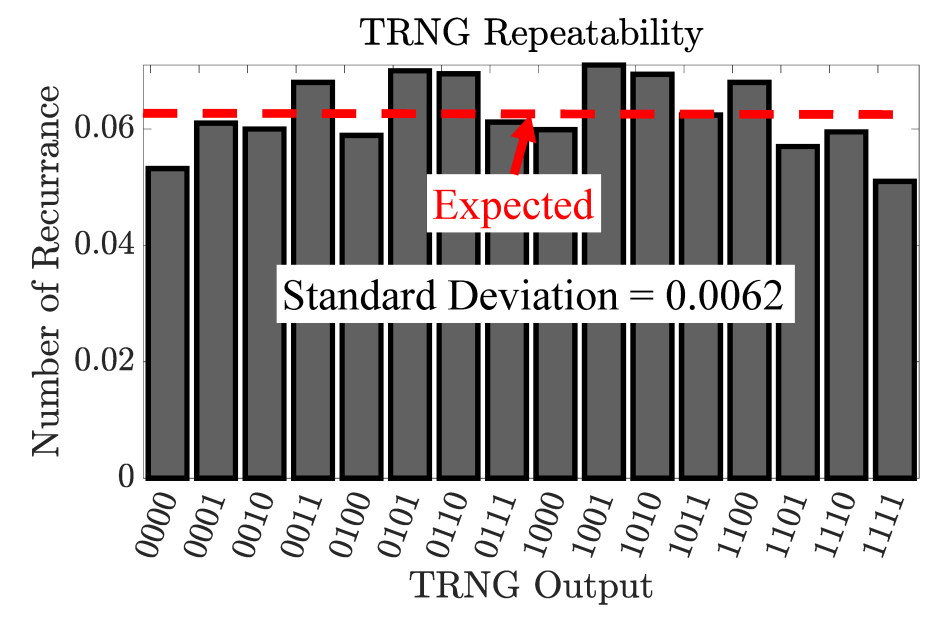

(b)

Figure 14. (a) Average entropy of all chips measured column-wise; (b) repeatability of TRNG outcomes out of 10,000 responses.

The energy, bandwidth and area of the proposed TRNG are found to be $0.41 \mathrm{pJ} / \mathrm{bit}$, $0.12 \mathrm{Gbit} / \mathrm{s}$ and $0.769 \mu \mathrm{m}^{2} /$ bit (including all peripheral circuits). We have also tested our TRNG outputs with NIST STS randomness test (summarized in Table 3). It is evident from the results that the proposed TRNG achieves excellent quality. Table 4 benchmarks the proposed TRNG with existing MRAM/STTRAM TRNG. We can observe that the proposed PUF is comparable to prior works and its energy (bandwidth) is significantly lower (higher) than others.

Table 3. Summary of NIST Suite Statistical Result.

\begin{tabular}{cccc}
\hline NIST Statistical Test & $\boldsymbol{p}$-Value & Proportion & Result \\
\hline Frequency & 0.349865 & $199 / 200$ & Pass \\
Block Frequency & 0.257217 & $199 / 200$ & Pass \\
Cumulative Sums & 0.393322 & $199 / 200$ & Pass \\
Discrete Fourier Transform & 0.476393 & $199 / 200$ & Pass \\
Approximate Entropy & 0.844361 & $200 / 200$ & Pass \\
\hline
\end{tabular}


Table 4. Performance comparison of different MRAM/STTRAM TRNG with proposed TRNG.

\begin{tabular}{ccccccc}
\hline TRNG & Correlation & Entropy & $\begin{array}{c}\text { Area }(\mathbf{M T J}) \\
\left(\mu \mathbf{m}^{2}\right)\end{array}$ & $\begin{array}{c}\text { Bandwidth } \\
(\mathbf{G b i t} / \mathbf{s})\end{array}$ & $\begin{array}{c}\text { Energy/bit } \\
(\mathbf{p J})\end{array}$ & Experimental \\
\hline$[20]$ & 0.003 & - & 0.014 & 0.0005 & 14.97 & Yes \\
\hline$[21]$ & - & - & $0.0085^{1}$ & $0.0833^{1}$ & $0.3386^{1}$ & Yes \\
\hline This Work & 0.05 & 0.95 & $0.769^{2}$ & 0.12 & $0.41^{2}$ & Yes \\
\hline
\end{tabular}

${ }^{1}$ Considering 3 ns of read time; ${ }^{2}$ Includes all peripheral circuitry and considers only 4 bits of each row. Thus, area is $2 \times$ of wPUF.

\section{Discussions}

$V_{D D}$ and temperature tracking: Any biasing technique to achieve a particular switching probability (pulse width/duration) is susceptible to $V_{D D} /$ temperature. Therefore, $V_{D D} /$ temperature tracking is required to select appropriate biasing condition which can be designed based on statistical data.

Considerations to other magnetic memory architecture: Consecutive writes of $1 \mathrm{~s}$ with less than $T_{1 \rightarrow 1}$ write time gives $75 \%, 50 \%$ and $35 \%$ of number of $1 \mathrm{~s}$ in the data (observation (c)) for the toggle MRAM chip. However, three different write times $\left(T_{75 \%} / T_{50 \%} / T_{35 \%}\right)$ can be implemented for other memory (that does not show this behavior).

Novelty of this work: Prior works consider bitwise normal distribution of switching probability. However, there are several practical challenges with the real memory implementation. First, it offers a narrow distribution with some columns/rows stuck at $0 / 1$. Besides, the real memory chip does not provide granular access to each individual bits and for that biasing of the bits cannot be bitwise tailored. Even custom biasing for each row is impractical. To the best of our knowledge, we make the first attempt to systematically understand and address these practical challenges in this paper.

Note that we have selected MRAM since it is very promising due to its low static and read power consumption. However, the proposed post-processing techniques to improve the inter-HD of PUF and entropy of TRNG are applicable to other memory technologies.

TRNG robustness to Machine Learning Attack: Random Number Generator (RNG) can be vulnerable to machine learning attack [30]. However, an RNG can be robust against such attack if the non-linearity is very high (i.e., no repetitive patterns in outcomes of RNG). Since the response of the proposed TRNG is non-linearly dependent on numerous parameters, e.g., write pulse width, write voltage and temperature due to non-linear magnetization dynamics of MRAM free layer, the proposed MRAM TRNG is expected to be robust against machine learning attack.

\section{Conclusions}

We have investigated TRNG and PUF implementation using magnetic memory and manipulation of write time and number of writes. We have analyzed the practical implications of designing TRNG/PUF using commercial MRAM and addressed these issues to achieve high quality.

Author Contributions: Conceptualization, M.N.I.K. and S.G.; methodology, M.N.I.K.; software, A.A.-S.; validation, M.N.I.K., C.Y.C., S.H.L. and A.A.-S.; formal analysis, M.N.I.K.; investigation, M.N.I.K.; resources, S.G.; data curation, C.Y.C. and S.H.L.; writing-M.N.I.K., A.A.-S. and S.G.; original draft preparation, M.N.I.K. and A.A.-S.; writing—review and editing, M.N.I.K., A.A.-S. and S.G.; visualization, M.N.I.K.; supervision, M.N.I.K. and S.G.; project administration, M.N.I.K. and S.G.; funding acquisition, S.G. All authors have read and agreed to the published version of the manuscript.

Funding: This work is supported by Semiconductor Research Corporation (2847.001 and 3011.001) and National Science Foundation (CNS-1722557, CCF-1718474, DGE-1723687, OIA-2040667 and DGE-1821766).

Conflicts of Interest: The authors declare no conflict of interest. 


\section{References}

1. Khan, M.N.I.; Bhasin, S.; Yuan, A.; Chattopadhyay, A.; Ghosh, S. Side-Channel Attack on STTRAM Based Cache for Cryptographic Application. In Proceedings of the 2017 IEEE International Conference on Computer Design (ICCD), Boston, MA, USA, 5-8 November 2017; pp. 33-40.

2. Lin, C.W.; Ghosh, S. Novel self-calibrating recycling sensor using Schmitt-Trigger and voltage boosting for fine-grained detection. In Proceedings of the Sixteenth International Symposium on Quality Electronic Design, Santa Clara, CA, USA, 2-4 March 2015; pp. 465-469.

3. Herder, C.; Yu, M.; Koushanfar, F.; Devadas, S. Physical Unclonable Functions and Applications: A Tutorial. Proc. IEEE 2014, 102, 1126-1141. [CrossRef]

4. Suh, G.E.; Devadas, S. Physical Unclonable Functions for Device Authentication and Secret Key Generation. In Proceedings of the 2007 44th ACM/IEEE Design Automation Conference, San Diego, CA, USA, 4-8 June 2007; pp. 9-14.

5. Tsoi, K.H.; Leung, K.H.; Leong, P.H.W. Compact FPGA-based true and pseudo random number generators. In Proceedings of the 11th Annual IEEE Symposium on Field-Programmable Custom Computing Machines, FCCM 2003, Napa, CA, USA, 9-11 April 2003; pp. 51-61.

6. Magnetic Tamper Detection Using Low-PowerHall Effect Sensors. Available online: http://www.ti.com/lit/ug/tidub69/tidub6 9.pdf (accessed on 12 January 2021).

7. Miura, N.; Fujimoto, D.; Tanaka, D.; Hayashi, Y.-I.; Homma, N.; Aoki, T.; Nagata, M. A local EM-analysis attack resistant cryptographic engine with fully-digital oscillator-based tamper-access sensor. In Proceedings of the 2014 Symposium on VLSI Circuits Digest of Technical Papers, Honolulu, HI, USA, 10-13 June 2014; pp. 1-2.

8. Ghosh, S.; Basak, A.; Bhunia, S. How Secure Are Printed Circuit Boards Against Trojan Attacks? IEEE Design Test 2015, 32, 7-16. [CrossRef]

9. Khan, M.N.I.; Nagarajan, K.; Ghosh, S. Hardware Trojans in Emerging Non-Volatile Memories. In Proceedings of the 2019 Design, Automation \& Test in Europe Conference \& Exhibition (DATE), Florence, Italy, 25-29 March 2019; pp. 396-401.

10. Khan, M.N.I.; De, A.; Ghosh, S. Cache-Out: Leaking Cache Memory Using Hardware Trojan. IEEE Trans. Very Large Scale Integr. (VLSI) Syst. 2020, 28, 1461-1470. [CrossRef]

11. Mazady, A.; Rahman, M.T.; Forte, D.; Anwar, M. Memristor PUF-A Security Primitive: Theory and Experiment. IEEE J. Emerg. Sel. Top. Circuits Syst. 2015, 5, 222-229. [CrossRef]

12. Iyengar, A.; Ghosh, S.; Ramclam, K.; Jang, J.-W.; Lin, C.-W. Spintronic PUFs for Security, Trust, and Authentication. J. Emerg. Technol. Comput. Syst. 2016, 13, 1-5. [CrossRef]

13. Ghosh, S.; Govindaraj, R. Spintronics for associative computation and hardware security. In Proceedings of the 2015 IEEE 58th International Midwest Symposium on Circuits and Systems (MWSCAS), Fort Collins, CO, USA, 2-5 August 2015; pp. 1-4.

14. Chen, A. Utilizing the Variability of Resistive Random Access Memory to Implement Reconfigurable Physical Unclonable Functions. IEEE Electron Device Lett. 2015, 36, 138-140. [CrossRef]

15. Zhang, L.; Fong, X.; Chang, C.; Kong, Z.H.; Roy, K. Highly reliable memory-based Physical Unclonable Function using SpinTransfer Torque MRAM. In Proceedings of the 2014 IEEE International Symposium on Circuits and Systems (ISCAS), Melbourne, VIC, Australia, 1-5 June 2014; pp. 2169-2172.

16. Chen, A.; Hu, X.S.; Jin, Y.; Niemier, M.; Yin, X. Using emerging technologies for hardware security beyond PUFs. In Proceedings of the 2016 Design, Automation \& Test in Europe Conference \& Exhibition (DATE), Dresden, Germany, 14-18 March 2016; pp. 1544-1549.

17. Das, J.; Scott, K.; Rajaram, S.; Burgett, D.; Bhanja, S. MRAM PUF: A Novel Geometry Based Magnetic PUF With Integrated CMOS. IEEE Trans. Nanotechnol. 2015, 14, 436-443. [CrossRef]

18. Vatajelu, E.I.; Natale, G.D.; Prinetto, P. Zero bit-error-rate weak PUF based on Spin-Transfer-Torque MRAM memories. In Proceedings of the 2017 IEEE 2nd International Verification and Security Workshop (IVSW), Thessaloniki, Greece, 3-5 July 2017; pp. 128-133.

19. Khaleghi, S.; Vinella, P.; Banerjee, S.; Rao, W. An STT-MRAM based strong PUF. In Proceedings of the 2016 IEEE/ACM International Symposium on Nanoscale Architectures (NANOARCH), Beijing, China, 18-20 July 2016; pp. 129-134.

20. Seki, A.F.T.; Kubota, K.Y.H.; Imamura, H.; Yuasa, S.; Ando, K. Spin dice: A scalable truly random number generator based on spintronics. Appl. Phys. Express 2014, 7, 083001.

21. Oosawa, S.; Konishi, T.; Onizawa, N.; Hanyu, T. Design of an STT-MTJ based true random number generator using digitally controlled probability-locked loop. In Proceedings of the 2015 IEEE 13th International New Circuits and Systems Conference (NEWCAS), Grenoble, France, 7-10 June 2015; pp. 1-4.

22. Fong, X.; Chen, M.; Roy, K. Generating true random numbers using on-chip complementary polarizer spin-transfer torque magnetic tunnel junctions. In Proceedings of the 72nd Device Research Conference, Santa Barbara, CA, USA, 22-25 June 2014; pp. 103-104.

23. Vatajelu, E.I.; Natale, G.D.; Prinetto, P. Security primitives (PUF and TRNG) with STT-MRAM. In Proceedings of the 2016 IEEE 34th VLSI Test Symposium (VTS), Las Vegas, NV, USA, 25-27 April 2016; pp. 1-4.

24. Khan, M.N.I.; Cheng, C.Y.; Lin, S.H.; Ash-Saki, A.; Ghosh, S. A Morphable Physically Unclonable Function and True Random Number Generator using a Commercial Magnetic Memory. In Proceedings of the 2020 21st International Symposium on Quality Electronic Design (ISQED), Santa Clara, CA, USA, 25-26 March 2020; p. 197. 
25. Basys3 ${ }^{\mathrm{TM}}$ FPGA Board Reference Manual. Available online: reference.digilentinc.com/_media/basys3:basys3_rm.pdf (accessed on 12 January 2021).

26. The Standard for Superior Measurements. Available online: https://www.keysight.com/us/en/assets/7018-04261/data-sheets/ 5991-3904.pdf (accessed on 12 January 2021).

27. Zhang, X.; Sun, G.; Zhang, Y.; Chen, Y.; Li, H.; Wen, W.; Di, J. A novel PUF based on cell error rate distribution of STT-RAM. In Proceedings of the 2016 21st Asia and South Pacific Design Automation Conference (ASP-DAC), Macau, China, 25-28 January 2016; pp. 342-347.

28. Zhang, L.; Fong, X.; Chang, C.; Kong, Z.H.; Roy, K. Optimizating Emerging Nonvolatile Memories for Dual-Mode Applications: Data Storage and Key Generator. IEEE Trans. Comput. Aided Des. Integr. Circuits Syst. 2015, 34, 1176-1187. [CrossRef]

29. Shannon, C.E. A mathematical theory of communication. Bell Syst. Tech. J. 1948, 27, 379-423. [CrossRef]

30. Kim, J.; Nili, H.; Truong, N.D.; Ahmed, T.; Yang, J.; Jeong, D.S.; Sriram, S.; Ranasinghe, D.C.; Ippolito, S.; Chun, H.; et al. Nano-Intrinsic True Random Number Generation: A Device to Data Study. IEEE Trans. Circuits Syst. Regul. Pap. 2019, 66, 2615-2626. 